\title{
Editorial
}

\section{Special issue: A Tribute to the Contributions to Plasma Physics of V. D. Shafranov: 1929-2014}

\author{
J. W. Connor ${ }^{1}$, V. P. Pastukhov ${ }^{2}$ and L. E. Zakharov ${ }^{3}$ \\ ${ }^{1}$ Euratom/CCFE Fusion Association, Culham Science Centre, Abingdon, Oxon OX143DB, UK \\ ${ }^{2}$ National Research Center "Kurchatov Institute", Moscow, Russia \\ ${ }^{3}$ LiW Fusion, PO Box 2391, Princeton, NJ 08543, USA
}

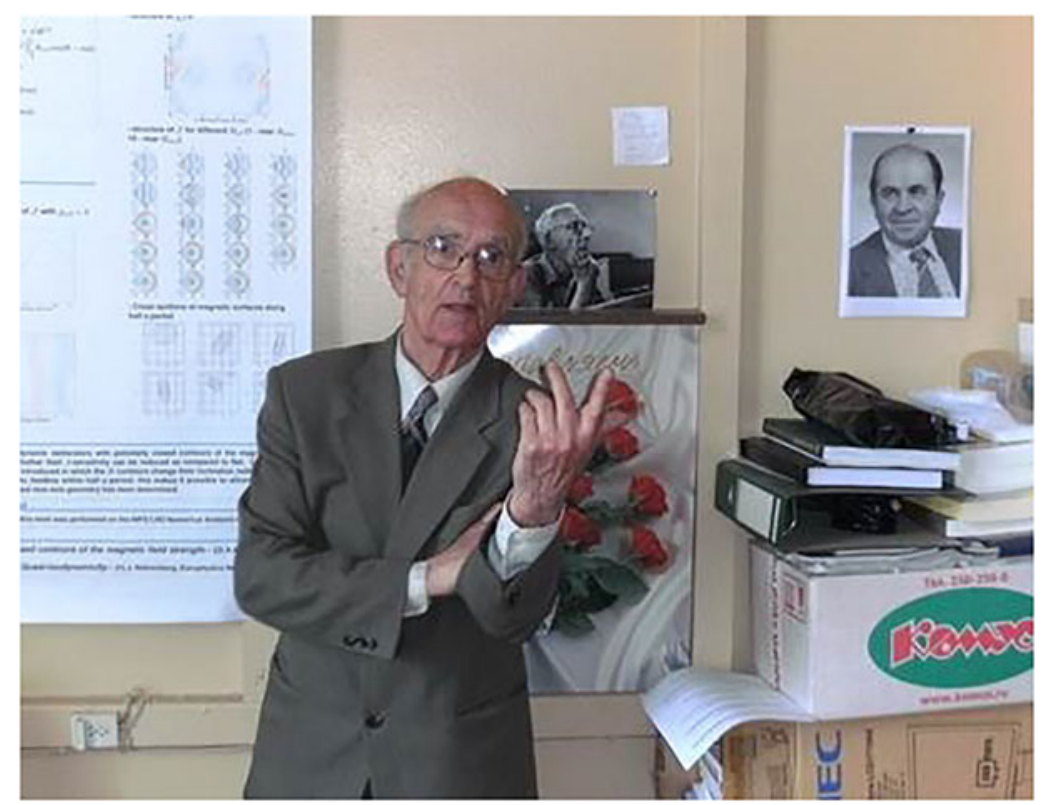

Academician Vitaly Dmitrievich Shafranov, who sadly died on 9 June 2014, is rightly regarded as one of the founding fathers of the theory of magnetic confinement fusion, his name being associated with many seminal aspects of plasma equilibrium and stability. For example, the Grad-Shafranov equation and the Shafranov shift are key concepts in axisymmetric toroidal plasma equilibrium theory, while the Kruskal-Shafranov condition is universally recognised as a fundamental limit on the toroidal plasma current, essential for maintaining macroscopic stability. He also derived elegant treatments of collisional diffusion in toroidal confinement systems. With Academician L. A. Artsimovich he introduced the concept of tokamaks with non-circular cross-sections which has led to the development of the present high-performance tokamak devices, such as JET, and to the poloidal divertors, necessary for the operation of a practical fusion device such as ITER. Later, he explored innovative non-axisymmetric equilibria. In addition he made contributions to basic plasma physics such as the theory of electromagnetic wave propagation and absorption and collisional shock waves in plasmas. 
V. D. Shafranov was born in 1929; after the Second World War he graduated from the Faculty of Physics of Moscow State University and moved to the Atomic Energy Institute, which later became known as the Kurchatov Institute. He joined the Theoretical Section under the leadership of Academician M. A. Leontovich, which was a part of the Plasma Research Division headed by L. A. Artsimovich (later succeeded by Academician B. B. Kadomtsev). Here his research career prospered, working fruitfully with a number of talented colleagues, leading to various seminal contributions as recalled above. Eventually he succeeded M. A. Leontovich as head of the Plasma Theory Department of the Institute of Nuclear Fusion, under B. B. Kadomtsev, a position he held for over 20 years (1981-2007). He was elected as a Corresponding Member of the USSR Academy of Sciences in 1981, and a Full Member of the Russian Academy of Sciences in 1997. Amongst the many honours he received was the Alfvén Prize of the European Physical Society in 2001. For approximately 30 years (1981-2011) he also played a most valued role as Editor-in-Chief of the journal Fizika Plazmy (Plasma Physics Reports), while he edited the series Reviews of Plasma Physics (founded by M. A. Leontovich) following the death of the previous Editor-in-Chief, B. B. Kadomtsev.

The papers assembled in this Special Issue to honour his memory, reflect many of the topics which he initiated. The paper, by Sergei Mirnov, a colleague at the Kurchatov Institute for many years, reviews aspects of the early Soviet era fusion research, and the key role Vitaly played in it. Four papers are concerned with issues of equilibrium and positional stability in axisymmetric systems. The paper by Peter Catto, Istvan Pusztai and Sergei Krasheninnikov provides solutions to a generalised Grad-Shafranov equation for global gravitational equilibria of rotating hot plasmas, with applications to accretion discs in white dwarfs. The one by Robert Dewar, Zensho Yoshida, Amitava Bhattacharjee and Stuart Hudson presents a novel approach to constructing equilibria using a variational formulation for relaxed magnetohydrodynamics (MHD) with a further generalisation to multiregion relaxed MHD. The two related papers by Jeff Friedberg, Antoine Cerfon, Jungpyo Lee and Martin Greenwald address the question of what is the maximum practical elongation in tokamak plasmas, taking into account the effect of the vertical stability feedback system.

There are two papers by Leonid Zakharov, a long-time disciple and close collaborator with Vitaly, which focus on the MHD stability issues central to Vitaly's interests. The first of these (with $\mathrm{Di} \mathrm{Hu}$ ) considers the quasilinear perturbed equilibria of resistively unstable current carrying plasmas; the second (with Calin Antanasiu, Karl Lackner, Erika Strumberger and Matthias Hoelzl) addresses the role of the currents flowing in the toroidal chamber wall when the tokamak plasma is in contact with it as a result of unstable kink modes during disruptions. A 3-D MHD equilibrium description of nonlinearly saturated ideal external kink/peeling mode structures in tokamaks is presented in the paper by W. A. Cooper, J. P. Graves, B. P. Duval, L. Porte, H. Reimerdes, O. Sauter and T.-M. Tran; this involves the computation of novel free boundary MHD equilibria with spontaneous 3-D deformations of the plasma vacuum interface. Vitaly was always alert to the necessity of comparing theory with experiment and the paper by Alan Turnbull and colleagues describes experimental results from the DIII-D tokamak on the external kink modes, so central to his work. The DIII-D device is a good example of the strongly shaped tokamaks that Vitaly and Lev Artsimovich espoused. The paper by Yu. V. Petrov, N. N. Bakharev, V. K. Gusev, V. B. Minaev, V. A. Kornev, G. S. Kurskiev, M. I. Patrov, N. V. Sakharov, S. Yu. Tolstyakov and P. B. Shchegolev addresses another instability in tokamaks, 
reporting the appearance of toroidal Alfvén eigenmodes (TAEs) in the GLOBUS-M spherical tokamak with neutral beam injection, and studies their impact on fast particle confinement.

Eventually Vitaly's interest moved from tokamaks to non-axisymmetric devices such as stellarators; advanced designs such as W7-X at IPP Greifswald, are descendants of an early novel proposal of Vitaly's: a set of linked mirrors called Dracon. The paper by Allen Boozer discusses the use of computation in designing such advanced stellarators. The one by Leonid Zakharov presents a numerical scheme for implementing the Hamada approach to constructing 3-D equilibria with nested magnetic surfaces. The paper by V. V. Nemov, S. V. Kasilov, W. Kernbichler and V. N. Kalyuzhnij is concerned with the collisionless loss of energetic particles in stellarator geometries in the presence of resonant perturbations of the magnetic field.

The recollections of one of us (L.E.Z.) provides further insights into Vitaly's scientific contributions: 'In 1951, Vitaly D. Shafranov, then only 21 , had written a report, later known as the paper: 'The stability of a flexible conductor in a longitudinal magnetic field'. His derivation was so clear that M. A. Leontovich, the head of plasma theory, agreed to be a co-author of the paper. This was exceptionable: M. A. hesitated to do this with the work of his staff members, even in cases when the guidance he had given to the authors was substantial. It was remarkable that the first stability condition of a toroidal plasma was obtained using a highly simplified theoretical model which still retains the key properties of free boundary plasmas. A non-physical flexible superconductor was considered instead of a plasma. Its skin current distribution was also unrealistic for a plasma, which is not superconducting. The formal result of the derivation was that a flexible cylinder with a current is always unstable. I am still puzzled that such a young person understood that the essence of the model is the presence of the $m / n=1 / 1$ kink mode, and that the criterion of its stabilization would also work for a toroidal plasma. This understanding of the special nature of the $m=1$ kink mode, which is insensitive to the current distribution, is stated firmly in the title: 'The stability' instead of a more cautions 'On some instabilities'. This paper by Shafranov and its various continuations complemented A. D. Sakharov's ideas of plasma confinement in a toroidal magnetic field with a plasma stability condition. With some delay, due to the difficulty in implementing the requirement of a strong toroidal field, the Shafranov criterion gave birth to tokamaks. The first one was built by N. A. Yavlinksi at the Kurchatov Institute. Another puzzle for me (and indeed for many others) was Shafranov's strong belief in ideal plasma stability conditions. He never paid attention to growth rates or to predictions of the temporal behaviour of MHD instabilities. An insight for this was later provided to me by Oleg Pogutse: the real plasma behaves like a superconductor because of very high electron thermal conductivity. It prevents the generation of a normal component of the magnetic field to the plasma surface and makes plasma much more stable than, e.g. liquid metal. Due to this high temperature plasma property, the derivation of ideal stability conditions is equivalent to the analysis of a perturbed equilibrium.

Equilibrium theory is the well-known tangible contribution of V. D. Shafranov to fusion. In the early 1960s he showed that equilibrium equations are valid essentially for any plasma in confinement devices, even in the presence of MHD fluctuations. The explanation of this was a very useful piece of education for me. Because of the importance of the equilibrium model for both tokamaks and stellarators, he paid attention to all details of equilibrium, including the requirements on external fields, conducting structures, magnetic diagnostics, positional stability, exact and asymptotic analytical solutions and the motivation for numerical simulations of equilibrium. I was 
amazed by his theory of equilibrium in stellarators with a spatially curved axis, where he introduced the complex curvature - an entirely new notion in differential geometry. Tokamaks with non-circular cross-section, suggested by Shafranov and Artsimovich in 1972, opened up the opportunity for enhanced confinement and the control of plasma-wall interactions by the use of poloidal divertors. Within ten years both these opportunities resulted in substantial progress in tokamak research.

All Shafranov's contributions to fusion were simple, transparent and fundamental - he liked simple interpretations. At the same time he liked to develop his ideas to completion and to be ready to use by others. It was a privilege to work with him and learn how not to be distracted by data in favour of trying to understand the basic plasma properties.'

Another of us (V. P.) recalls Vitaly's abilities as a poet. 'All his colleagues in Russia and many of his colleagues abroad are familiar with his poetic talent. Poetry was a very important part of his life, even from his youth. Vitaly wrote many rhymes, and even long poems, about his colleagues, friends and memorable events in the fusion community. All these rhymes and poems are full of latent humour and friendly jokes. He was the life and soul of many performances in the Kurchatov Institute and wrote numerous rhymes and songs for them. In 2009 Vitaly's colleagues collected and published a book of his rhymes and poems entitled 'Non-Scientific Proceedings' (in Russian). The book begins with an epic poem, OPIADA, which was written in the style of Homer's Iliad and devoted to the 50th Birthday of L. A. Artsimovich (in 1959). Here, OPI is the Russian abbreviation for the Plasma Research Division, headed by L. A. Artsimovich. The next remarkable long poem in the book is 'History of OPI's Fusion from the beginning through modern days', written in 1967. These comic verses describe the early stage of fusion research in Russia, including the first historic visit of British scientists to the Kurchatov Institute and their measurements of a high electron temperature in the tokamak T-3A. In 1978 Shafranov staged a musical performance 'Opera TOKAMANSCHINA', devoted to the 50th birthday of B. B. Kadomtsev. In this case Vitaly used the style of the classic opera KHOVANSCHINA, composed by M. P. Moussorgsky. We hope that this brief memoir will help our readers to understand more fully the breadth and brilliance of Shafranov's great talents.'

In addition to Vitaly's scientific contributions and poetic talents recalled above, one should not forget his warm and friendly personality, always supportive of colleagues. J. W. C. recalls the kindness and hospitality of Vitaly when he visited the Kurchatov institute with Dr Christopher Watson under an Anglo-Soviet Scientific Collaboration Agreement in 1975. Not only did he ensure that we had an enjoyable cultural experience, but he taught us the value of using Hamada coordinates in a joint paper (but one largely written by him!) on classical collisional diffusion in non-axisymmetric devices.

We trust this volume of contributions will help to preserve the memory of one of the true founders of magnetic confinement theory: V. D. Shafranov (1929-2014). 